\title{
One Sample Image Recognition Algorithm based on Improved Sub-pattern Principle Component Analysis
}

\author{
${ }^{1}$ Li yong-qiang and ${ }^{2}$ PanJin \\ ${ }^{1}$ (Software Technology Vocational College, North China University of Water \\ Resources and Electric Power, Zhengzhou 450045, China) \\ ${ }^{2}$ (Henan Procuratorial Vocational College Zhengzhou 451191, China) \\ 1696450309@qq.com ${ }^{1}, 1632921040 @ q q$. com $^{2}$
}

\begin{abstract}
In order to improve the recognition performance of one sample image per person, this paper proposed a one sample image recognition algorithm based on improved sub-pattern principle component analysis. Firstly, the face is divided into some blocks, and Sub-pattern PCA is used to extract features, and then information entropy is used to assign proper weights of blocks features, finally, support vector machine is used recognize the face and the experimental results are carried out on some face data. The results demonstrate that the proposed algorithm has improved the recognition rate and speed of face, and has robust.
\end{abstract}

Keywords: single sample; face recognition; eigenface; sub-pattern principle component analysis

\section{Introduction}

Human face is exclusive, non-contact and non-reproduced. It is the most common biological characteristics, which is widely applied in safety protection, recognition, video retrieval and other fields [1]. To solve the human face recognition issues, many scholars have spent a lot of time and efforts studying, and put forward many human face recognition algorithms [2]. However, in the forensics, passport verification and other special occasions, every class of human face can only get one piece of human face image, which is a typical single-sample human face recognition problem. Therefore, how to improve the correct rate of single-sample human face recognition has become a hot issue in current research [3].

In the process of single-sample human face recognition, feature extraction is the most critical step, and it is essential to extract as much feature information as possible from a single human face image [4]. Literature [5] proposed to adopt the single-sample human face recognition method with virtual human face technology, which had improved the accuracy of human face recognition, but it had relatively high computational complexity, and thus it is difficult to meet the real-time human face recognition requirements; Literature [6] comprehensively considered the principal component analysis and integral projection features to complement each other, extract human face features and enhance the human face recognition rate, but the principal component analysis was a linear dimension reduction method, so it was easy to lose some detailed feature information; Literature [7] proposed block-based single-sample human face recognition algorithm, used the linear analysis algorithm to select important features, and obtained ideal recognition results; Literature [8] proposed the single-sample human face recognition for Eigenfaces, but the algorithm only extracts the global features from human face images, which was rather sensitive to changes in light, posture and expression; Literature [9] proposed a SpPCA-based human face recognition algorithm, first divided the human face 
images into multiple sub-models, extracted the human face features from each sub-model, and finally spliced the sub-model feature together to realize the human face recognition. The algorithm had integrated the advantages of global and local features, and thus had better robustness to light changes. Due to the different roles of varied human face parts in human face recognition results, for example, compared to the forehead and other parts, nose, mouth and eyebrows are more important [10-11]. However, in practical applications, SpPCA algorithm has ignored this, and the recognition performance needs to be improved [12].

In order to improve the performance of single-sample human face recognition, this paper proposes a ISpPCA-based single-sample human face recognition algorithm, and adopts multiple face database to test the human face recognition effects of the presented algorithm.

\section{ISpPCA-based Single-sample Human Face Recognition Algorithm}

\subsection{Human Face Recognition Process}

The basic steps of ISpPCA-based human face recognition algorithm are:

(1) Evenly divide single human faces into multiple sub-blocks, namely, sub-models, and adopt PCA to obtain the projection vector of each sub-block.

(2) Weigh for each sub-block and connect them into human face feature vector.

(3) Adopt human face classifier to make decisions towards the human face images and output the recognition results,

The single-sample face recognition process is shown in Figure 1.

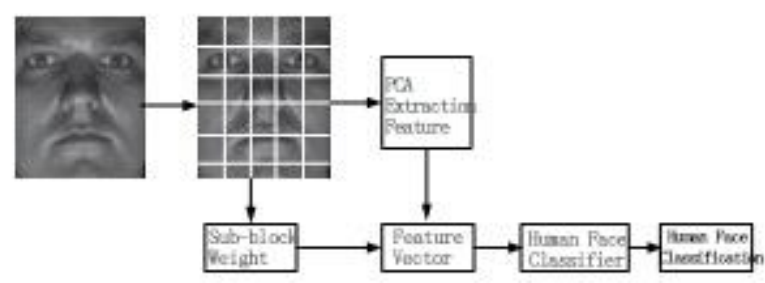

Figure 1. Working Process for Human Face Recognition Algorithm

\subsection{Human Face Image is Divided into Multiple Sub-models}

(1) Assumed the size of human face image is $\mathrm{M} \times \mathrm{N}$.

(2) Divide human face images into L pieces of sub-images with equal size, and the specific results are shown in Figure 2.

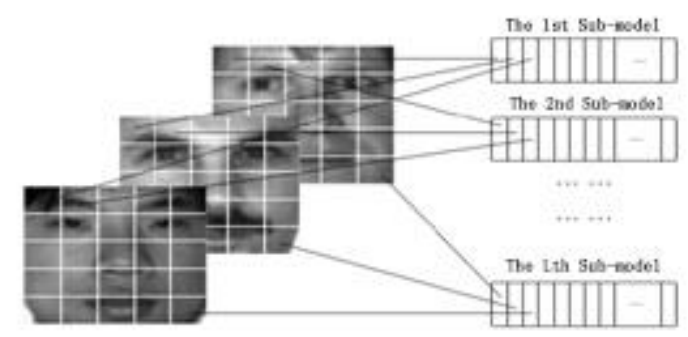

Figure 2. Division of Human Face Sub-model

\subsection{Principal Component Analysis for Feature Extraction}

Through the above steps, the single-sample human face image can be described by the the following matrix: 


$$
I=\left[\begin{array}{cccc}
I_{11} & I_{12} & \cdots & I_{1 q} \\
I_{21} & I_{22} & \cdots & I_{2 q} \\
\cdots & \cdots & \cdots & \cdots \\
I_{p 1} & I_{p 2} & \cdots & I_{p q}
\end{array}\right]
$$

All sub-models in the training set of human face images build a new training subset, namely:

Where, $j=1,2, \cdots, L$.

$$
A_{j}=\left[I_{1 j}, I_{2 j}, \cdots, I_{K j}\right]
$$

Assumed the $j$-th sub-model divergence matrix is:

$$
S_{j}=\frac{1}{K} \sum_{i=1}^{K}\left(I_{i j}-\bar{A}_{j}\right)\left(I_{i j}-\bar{A}_{j}\right)^{T}
$$

Where, $\bar{A}_{j}=\frac{1}{K} \sum_{i=1}^{K} I_{i j}$.

By adopting the matrix feature value decomposition method, this paper solves the previous $M^{\prime}$-th feature vector in $S_{j}$, namely

$$
S_{j} \Phi_{j}=\Phi_{j} \Lambda_{j}
$$

Where, $\Lambda_{j}$ stands for diagonal matrix; $\Phi_{j}=\left[\phi_{j 1}, \phi_{j 2}, \cdots, \phi_{j M^{\prime}}\right]$.

Then the projection vector of the ${ }^{i}$-th human face's ${ }^{j}$-th sub-block is:

$$
S p_{i j}=\Phi_{j}^{T} \cdot\left(I_{i j}-A_{j}\right)
$$

Where, $i=1, \ldots, K, j=1, \ldots, L$.

Thus, the feature value of ${ }^{i}$-th human face image can be described as:

$$
S p_{i}=\left[S p_{i 1}, S p_{i 2}, \cdots, S p_{i L}\right]
$$

By adopting the above similar steps, the projection vector of the $j_{\text {-th }}$ sub-block can be obtained:

$$
S p_{\text {test } j}=\Phi_{j}^{T} \cdot\left(I_{\text {test } j}-\bar{A}_{j}\right)
$$

At last, the test human face feature vector can be described as:

\subsection{Sub-block Weight}

$$
S p_{\text {test }}=\left[S p_{\text {test } 1}, S p_{\text {test } 2}, \cdots, S p_{\text {test } L}\right]
$$

Each sub-block has different positions in human face and makes different contributions to human face recognition result, so it can distinguish by giving a certain weight to each sub-block. The information entropy is:

$$
H(x)=\sum_{i=1}^{s} p_{i} \log \left(\frac{1}{p_{i}}\right)=-\sum_{i=1}^{s} p_{i} \log \left(p_{i}\right)
$$

For each sub-block image, the information entropy is:

$$
\omega_{i}=-\sum_{j=1}^{s} p\left(x_{i}\right) \log \left(p\left(x_{i}\right)\right)
$$

Where, s represents the gray level of sub-block; $p(x i)$ represents the probability of the xi-th pixel occurrence.

\subsection{Human Face Classifier}

Assumed human face image data set $\{x i, y i\}, x i \in R n, i=1,2, \ldots, n$, xi represents the selected human face feature vector; yi represents the human face category, and the classification plane can be described as:

$$
y=\omega^{T} \Phi(x)+b
$$

Where, $\omega$ and $\mathrm{b}$ stand for parameters.

By introducing the relaxation factor, the formula is transformed into: 


$$
\begin{aligned}
& \min J(w, \xi)=\frac{1}{2}\|w\|^{2}+c \sum_{i=1}^{n} \xi_{i} \\
& \text { s.t. } \\
& y_{i}\left(w \sqsubset \Phi\left(x_{i}\right)+b\right) \geq 1-\xi_{i} \\
& \quad \xi \geq 0, i=1,2 \cdots, n
\end{aligned}
$$

Where, $\mathrm{C}$ is the penalty parameter.

The binary-class function of human face image is transformed into:

$$
f(x)=\operatorname{sgn}\left(\alpha_{i} y_{i} \Phi\left(x_{i}\right) \cdot \Phi(x)+b\right)
$$

Where, $\Phi\left(x_{i}\right) \llbracket \Phi\left(x_{j}\right)$ represents the dot product.

By introducing the kernel function over non-linear human face, the following equation can be got:

$$
f(x)=\operatorname{sgn}\left(\alpha_{i} y_{i} k\left(x, x_{i}\right)+b\right)
$$

\section{Simulation Experiments}

To test the performance of presented single-sample human face recognition algorithm, this paper selects Yale B, ORL, PIE human face database to make simulation experiments, achieves the human face recognition algorithm by using Matlab 2009 software programming, adopts PCA and SpPCA under the same conditions for comparative experiment, and evaluates advantages and disadvantages of the algorithm via human face recognition rate.

\subsection{Yale B Human Face Recognition}

Yale B human face contains images of 38 persons under 64 different lighting conditions, and each person has 5 sub-sets under different lighting condition. Subset 1 includes 7 images, and sub-sets 2, 3, 4 and 5 respectively contain 12, 12, 14 and 19 images [13]. Yale B human face images are shown in Figure 3.

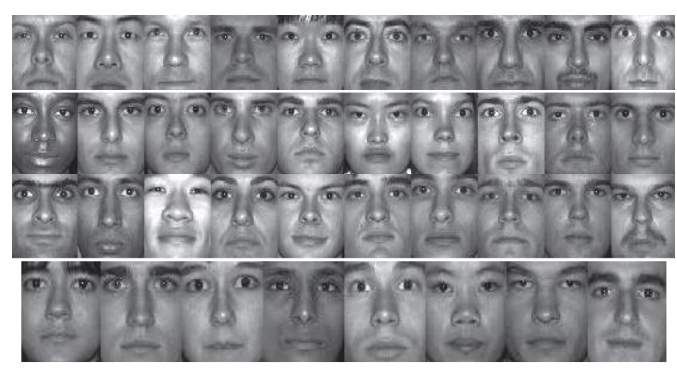

Figure 3. Human Face Images

For the human face recognition rate and rejection rate of different algorithms, refer to Figure 4. According to Figure 4, under different light conditions, the recognition rate of ISpPCA algorithm has been improved significantly, and the rejection rate is relatively small. Besides, the greater the light changes are, the more obvious advantages the proposed method has. The results show, ISpPCA algorithm has good robustness to light changes, and has solved the single-sample human face recognition problems under light changes. 


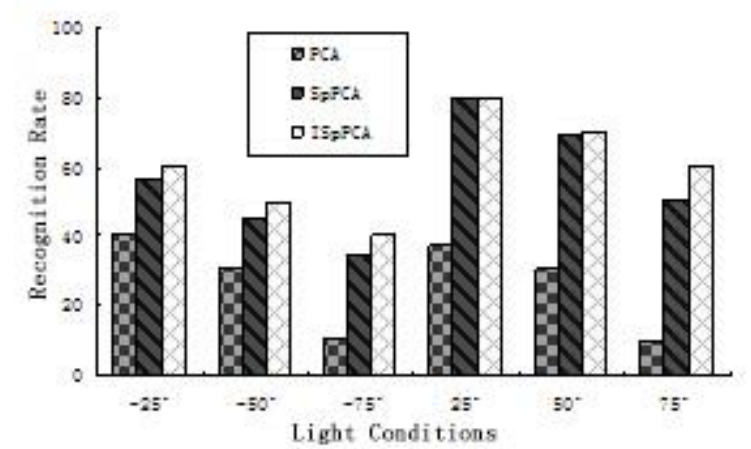

Figure 4. Yale B Human Face Recognition Rate

\subsection{ORL Human Face Recognition}

ORL human face database contains 40 classes of faces, each class has 10 images, and there are a total of 400 images [14]. Parts of human face sample images in ORL human face database are shown in Figure 5.

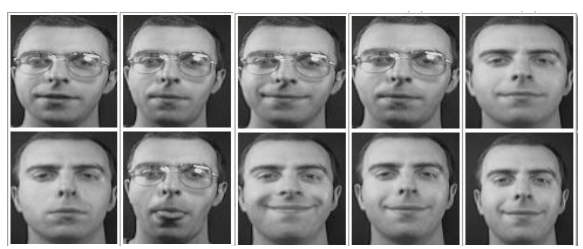

Figure 5. Sample Images of ORL Human Face Database

This paper selects the first image as a training sample, uses PCA, SpPCA and ISpPCA algorithms to extract features from ORL human face database, adopts the support vector machine to build human face classifier, selects the rest images as testing samples, and the results obtained are shown in Figure 7. According to Figure 6, compared to PCA, SpPCA and ISpPCA algorithms, human face recognition rate has been improved, which is mainly because that the use of sub-model idea can accurately extract the information structure from human face images, ISpPCA algorithm has better stability, and the comparison results prove the effectiveness and superiority of ISpPCA algorithm.

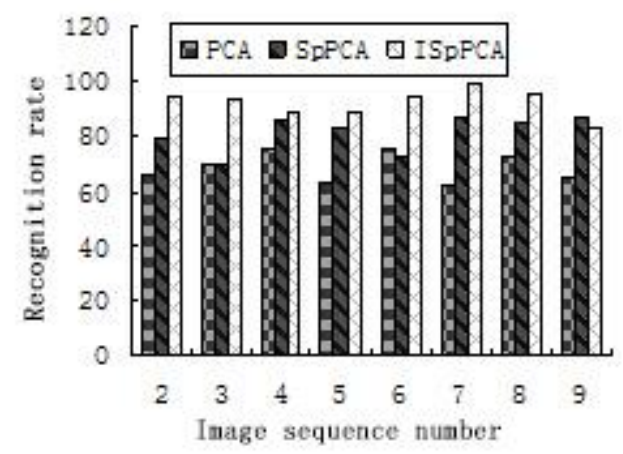

Figure 6. ORL Human Face Recognition Rate

\subsection{PIE Human Face Recognition}

Human face database PIE contains 68 persons, each person has about 170 images, including 135 images of training data, and the rest images are used as test data. The image 
was taken in different light, time, angle, facial expressions, gestures and other conditions, and the human face image examples are shown in Figure 7 [15].

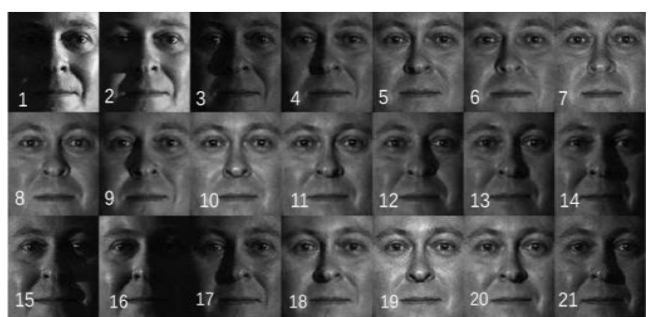

\section{Figure 7. A class of Human Face Image in PIE Human Face Database}

The PIE human face database recognition results of PCA, SpPCA and ISpPCA algorithms are shown in Figure 8. For PCA and SpPCA algorithms, ISpPCA algorithm shows obvious advantages, and the comparative results proved the superiority of ISpPCA algorithm once again.

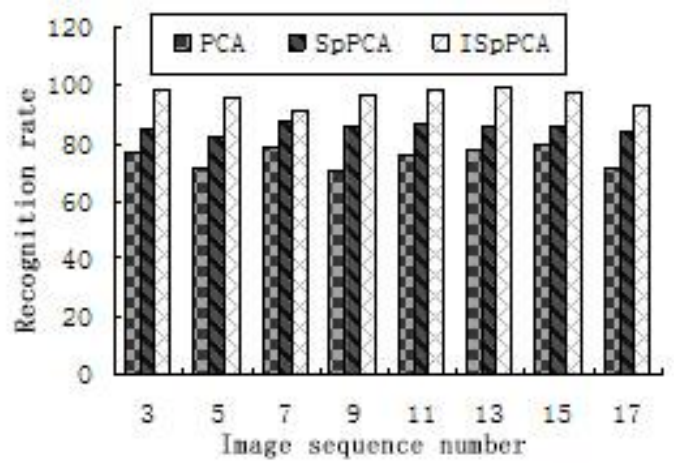

Figure 8. PIE Human Face Recognition Rate

\section{Conclusion}

To solve the current single-sample human face recognition problems, this paper proposes an ISpPCA single-sample human face recognition algorithm. The results show that ISpPCA algorithm has good robustness over light changes, has significantly improved the feature facial methods, makes up the deficiencies of SpPCA algorithm, improves the single-sample human face recognition rate, and enhances the efficiency of human face recognition and robustness.

\section{References}

[1] K C. Lee, J. Ho and D. Kriegman, "Acquiring linear subspaces for face recognition under variable lighting [J]", IEEE Transactions on Pattern Analysis and Machine Intelligence, vol. 27, no. 5, (2005), pp. 684-698.

[2] Y. Xu, A. Zhong and J. Yang, "LPP solution schemes for use with face recognition [J]", Pattern Recognition, vol. 43, no. 12, (2010), pp. 4165-4176.

[3] Y. Xu, F. Song and G. Feng, "A novel local preserving projection scheme for use with face recognition [J]”, Expert Systems with Applications, vol. 37, no. 9, (2010), pp. 6718-6721.

[4] V. Vishwakarma, S. Pandey and M. Gupta, "An illumination invariant accurate face recognition with down scaling of DCT coefficients [J]", Journal of Computing and Information Technology, vol. 18, no. 1, (2010), pp. 53-67.

[5] M. Koc and A. Barkana, "A new solution to one sample problem in face recognition using FLDA [J]", Applied Mathematics and Computation, vol. 217, no. 24, (2011), pp. 10368-10376.

[6] S. Chen and Y. Zhu, "Sub-pattern based principle component analysis [J]", Pattern Recognition, vol. vol. 37, no. 5, (2004), pp. 1081-1083. 
[7] K. Tan and S. Chen, "Adaptively weighted sub-pattern PCA for face recognition [J]", Neurocomputing, vol. 64, no. 12, (2005), pp. 505-511.

[8] R. Zhao, B. Fang and J. Wen, "Face recognition with single training sample per person based on adaptive weighted LBP [J]", Computer Engineering and Applications, vol. 48, no. 31, (2012), pp. 146-149.

[9] Q. Hong, S.-C. Chen and X.-L. Ni, "Sub-Pattern Canonieal Correlation Analysis with APPlieation in Faee Recognition [J]”, ACTA AUTOMATICA SINICA, vol. 43, no. 1, (2008), pp. 21-30.

[10] S. Zhao, Z. Zhang and P. Zhang, "Enhanced CCA and its Applications in Feature Fusion of Face Recognition [J]", Journal of Computer-aided design \& Computer graphi, vol. 21, no. 3, (2009), pp. 394-400.

[11] P. C. Hsieh and P C. Tung, "A novel hybrid approach based on sub-pattern technique and whitened PCA for face recognition [J]”, Pattern Recognition, vol. 42, no. 5, (2009), pp. 978-984.

[12] L. Tang, R. Xiong and J. Chu, "Weighted Modular 2DPCA-Based Face Recognition from a Single Sample Image Perclass [J]", Journal of Image and Graphics, vol. 13, no. 12, (2008), pp. 2307-2313.

[13] X.-z. Wang, Liyan and Guo-lihon, "Face recognition algorithm based on BD-PCA and K-NN [J]", Journal of PLA University of Science and Technology (Natural Science Editon), vol. 11, no. 6, (2010), pp. 623-238.

[14] J. Zhang and L. Liyuan, "Multi-view face recognition based on factor analysis and sparse representation, Computer [J]", Computer Engineering and Applications, vol. 49, no. 5, (2013), pp. 154-159.

[15] Y L. Zhu, "Sub-pattern non-negative matrix factorization based on random subspace for face recognition [C]", In: International conference on wavelet analysis and pattern recognition, (2007), pp. $1356-1360$.

[16] J. Y. Wu, W. J. Li, J. P. Huang, J. L. Zhang and D. R. Chen, "Key techniques for Mobile Internet: a survey, SCIENTIA SINICA Informationis, vol. 45, no. 1, (2015), pp. 45-69.

[17] J. Zhang and J.-y. Wu, "Provable Secure Efficient Arbitrated Quantum Signature Scheme [J]", Journal of Beijing University of Posts and Telecommunications, vol. 36, no. 2, (2013), pp. 113-116.

\section{Authors}

Li Yongqiang (1974.1-) Lecturer, Master, and Research Orientation: Computing Network;

Pan Jin (1974.11-) Associate Professor, Master, Research Orientation: Computer Network 
International Journal of Signal Processing, Image Processing and Pattern Recognition Vol.8, No.9 (2015) 